\title{
New Integrated TBM Disc Cutter Deviation Analysis And Design of The Supporting Cutter-Changing Robot End-Effector
}

Hao Chen ( $\sim 2538110473 @ q q . c o m$ )

Dalian University of Technology

Hao Li

Dalian University of Technology

Junzhou Huo

Dalian University of Technology

Bowen Yang

Dalian University of Technology

Fan Yang

Dalian University of Technology

\section{Research Article}

Keywords: TBM, Integrated disc cutter, Assembly deviation analysis, Cutter-changing robot end-effector

Posted Date: January 28th, 2022

DOI: https://doi.org/10.21203/rs.3.rs-1200920/v1

License: (c) (i) This work is licensed under a Creative Commons Attribution 4.0 International License.

Read Full License 


\title{
New Integrated TBM Disc cutter Deviation Analysis and Design of the
}

\section{Supporting Cutter-Changing Robot End-Effector}

\author{
Hao Chen ${ }^{1 *}$, Hao Li ${ }^{1}$, Junzhou Huo ${ }^{1}$, Bowen Yang ${ }^{1}$, Fan Yang ${ }^{1}$
}

\begin{abstract}
At present, the replacement of disc cutters of the tunnel boring machine (TBM) is a very important and difficult manual operation. Therefore, it is meaningful to study disc cutters of TBM replacement with a robot. In this paper, the machining and assembly deviation of the new integrated disc cutter developed by our research group is modeled and analyzed by using the improved Jacobian-Torsor model, and the analysis results are verified by experiments. On this basis, aiming at the possible radial deviation and angle deviation in the working process of a robot replacing TBM disc cutters, a cutter-changing robot end-effector composed of a guiding mechanism, a flexible connecting mechanism and a universal wrench is designed for the disassembly and assembly of the new integrated disc cutter, and scaled-down models were made for the experiment. The test results show that when there is a radial deviation of $19.21 \%$ or an angle deviation of $11^{\circ}$ between the fastening bolt and the end-effector of the robot, the robot end-effector can complete the docking with the fastening bolt.
\end{abstract}

Keywords: TBM, Integrated disc cutter, Assembly deviation analysis, Cutter-changing robot end-effector

\section{Introduction}

The tunnel boring machine (TBM) is the main equipment for tunnel construction at present. The disc cutters installed on the cutter head of TBM are the direct rock-breaking tool, which needs to be replaced frequently. At present, the disc cutters are mainly replaced manually, which is dangerous, inefficient and difficult. The rise of the robot industry provides a new way for replacing TBM disc cutters. Since Bouygues company[1] of France first proposed to use robots to replace disc cutters for TBM in 2007, researchers in Germany, Japan, China, and other countries have successively developed robots to replace disc cutters for TBM in the following ten years[2-4]. However, most of them are experimental products, and the working effect of a few products applied in practical engineering can not meet the actual needs[4].

The application of integrated disc cutters is the premise for the robot to replace the disc cutters for TBM. The purpose of this paper is to design the end- effector of the tool-changing robot for an integrated ${ }^{1}$ disc cutter developed by our research team. The main function of the robot is to dock and twist the fastening bolts of the integrated disc cutter.

Docking mechanisms are mostly used in aerospace and underwater technology fields. Common docking mechanisms include rod-cone typed docking mechanism[5], the androgynous peripheral docking mechanism[6], low impact docking system[7], etc. In this paper, the improved rod-cone typed docking mechanism is used to design the docking mechanism of the cutter-changing robot end-effector.

Research on using the robot to assemble and disassemble bolts. Chu, B.[8], etc. designed a bolt connection device for steel beam assembly on the construction site. The robotic bolting device consists of a bolting end-effector, and a gantry-type robotic manipulator, which places the bolts to each bolting position. Zhang, Q.[9], etc. designed a bolt-screwing tool based on a pneumatic slip ring structure, which

\footnotetext{
* Correspondence:2538110473@qq.com

${ }^{1}$ School of Mechanical Engineering, Dalian University of Technology, Dalian, 116024, China

Full list of author information is available at the end of the article
} 
can realize two-DOF motion of clamping-releasing and rotating. The tool consists of a pneumatic slip ring with a sealed structure and a cylinder-driven gripper. Li, R.Y.[10], etc. presents a novel spiral search technique developed to improve the rate of successful engagement between the robot end-effector and the screw heads despite uncertainties in the location of the screws. DiFilippo, N. M., and Jouaneh, M. K.[11] introduced a robotic system that combines force and visual sensors to help remove screws from laptops. Their work focuses on building and testing computer vision modules that automatically find screws. Zhang Q.[12], etc. proposed a novel approach for flexible manipulator conducting screwing task based on robotenvironment contact classification. They used the logistic regression method to classify the contact state according to the force signal, to judge whether the bolt is tightened.

Most of the above studies are aimed at the design of the robot end-effector or the development of the related control strategies and computer vision algorithms, and there is no deviation analysis of the robot's working object. However, the applicability of the robot endeffector is an important factor affecting the performance of the robot. The targeted design of the robot end-effector according to the deviation analysis results of the robot's working object can greatly improve the applicability of the robot.

The 3-D tolerance analysis technology is very suitable for the deviation analysis of complex assembly[13, 14]. Common 3D tolerance analysis models include Small Displacement Torsor model[1517], T-Map model [18, 19], Matrix model[20], Unified Jacobian-Torsor model, SDT[21-23] and DLM (Direct Linearization Method) [13, 24].

The Jacobian-Torsor model combines the small displacement spinor theory and the Jacobian matrix in robotics. Through the mathematical representation of the change of geometric elements by the small displacement spinor, the deviation of functional elements is transmitted by the Jacobian matrix, to be transformed into the change of functional requirements. In 2016, Zeng, W.H.[23] proposed an improved
Jacobian-Torsor model, which considers the case with complex transmission paths such as local parallel. On this basis, this paper analyzes the deviation of the new integrated TBM disc cutter system. According to the deviation analysis results of the integrated disc cutter system, the matching cutter-changing robot endeffector is designed.

\section{New integrated TBM disc cutter}

Traditional disc cutter has many unconnected parts, and it is difficult for robots to disassemble and assemble. To realize rapid cutter change by the robot, an integrated disc cutter suitable for robot operation should be designed first. To this end, an integrated disc cutter was designed. The disc cutter is mainly composed of 1 cutter box, 2 cutter holders, 1 disc cutter, 2 clamping blocks, 2 cushion blocks, 2 shift forks, 2 lifting rods, 2 thread fastening systems, etc., as shown in Fig. 1.

The fastening bolt is installed on the cutter holder through bearings, the fastening bolts and the shift forks are connected by threads, the clamping blocks and the shift forks are hinged, and rotating the fastening bolts can make the clamping blocks swing, as shown in Fig. 2. Rotate the fastening bolts to drive the shift forks to move upward along the fastening bolts, and then push the clamping blocks to swing outwards until they are tightly pressed into the grooves on the cutter holder, and the disc cutter is fixed on the cutter head. Reverse rotation of the fastening bolts can remove the disc cutter from the cutter head.

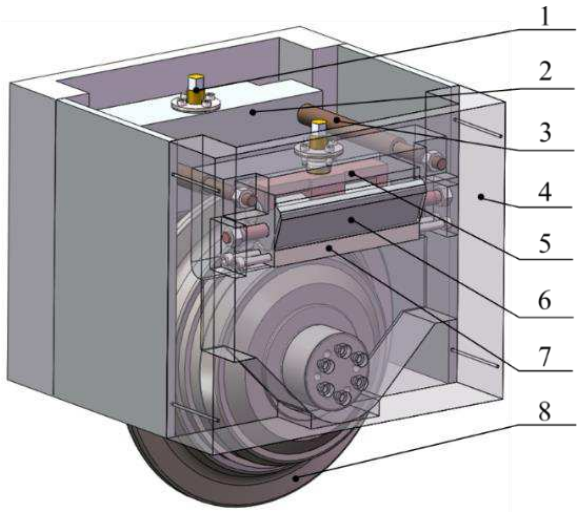

1- Thread fastening system, 2- Cutter holder, 3- Lifting rod, 4Cutter box, 5- Shift fork, 6-Clamping block, 7-Cushion block, 8- Disc cutter

Fig. 1 New integrated TBM disc cutter 


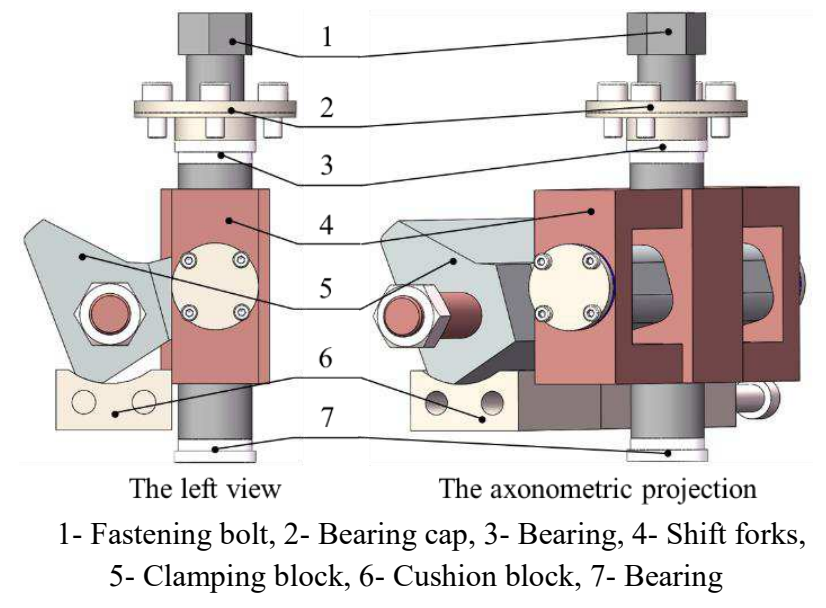

Fig. 2 Thread fastening system

\section{Deviation analysis of new integrated TBM disc cutter}

Position deviation of fastening bolts of the new integrated disc cutter system is the main source of the deviation in the connection of the robot end-effector and the disc cutter fastening bolt. Accurate calculation of the position deviation of the fastening bolts is the basis for the structural design of the cutter-changing end-effector. The deviation sources affecting assembly accuracy include: deviation of geometric location and orientation, variation of geometric form and deviation of part location and orientation[25].

The position deviations of the two fastening bolts of the new integrated disc cutter system mainly come from the following five parts. Assembly deviation of the threaded fastening system, $F R_{\mathrm{b}}$. The assembly deviation between the cutter holders and the disc cutter, $F R_{\mathrm{t}}$. The dimensional deviation of the cutter holders and the disc cutter in the direction of the cutter axis, $A_{0}$. The installation deviation between the cutter holders and the cutter box, $F R_{\mathrm{h}}$. The welding deviation between the cutter box and the mounting hole of the cutter head, $N$. Therefore, the position deviations of the fastening bolts can be described as Eq. (1).

$$
E=F R_{b}+F R_{t}+A_{0}+F R_{h}+N
$$

The deviation $F R_{\mathrm{b}}$ can be expressed as:

$$
F R_{b}=\left[\begin{array}{llllll}
u_{1} & v_{1} & w_{1} & \alpha_{1} & \beta_{1} & \gamma_{1}
\end{array}\right]^{T}
$$

Where, $u, v$, and $w$ are the translation vector parameters of the axes $x, y$, and $z$ respectively. $\alpha, \beta, \gamma$ are the rotation vectors of the axis $x, y$, and $z$ respectively. The same goes for the following.

The deviation $F R_{\mathrm{t}}$ can be expressed as:

$$
F R_{t}=\left[\begin{array}{llllll}
u_{2} & v_{2} & w_{2} & \alpha_{2} & \beta_{2} & \gamma_{2}
\end{array}\right]^{T}
$$

The deviation $\mathrm{A}_{0}$ can be expressed as:

$$
A_{0}=\left[\begin{array}{llllll}
0 & 0 & w_{3} & 0 & 0 & 0
\end{array}\right]^{T}
$$

The deviation $F R_{\mathrm{h}}$ can be expressed as:

$$
F R_{h}=\left[\begin{array}{llllll}
0 & v_{4} & 0 & 0 & 0 & \gamma_{4}
\end{array}\right]^{T}
$$

The deviation $N$ can be expressed as:

$$
N=\left[\begin{array}{llllll}
0 & 0 & 0 & 0 & \beta_{5} & \gamma_{5}
\end{array}\right]^{T}
$$

In summary, the position deviation of the fastening bolts is:

$$
E=\left[\begin{array}{c}
u \\
v \\
w \\
\alpha \\
\beta \\
\gamma
\end{array}\right]=\left[\begin{array}{c}
u_{1}+u_{2} \\
v_{1}+v_{2}+v_{4} \\
w_{1}+w_{2}+w_{3} \\
\alpha_{1}+\alpha_{2} \\
\beta_{1}+\beta_{2}+\beta_{5} \\
\gamma_{1}+\gamma_{2}+\gamma_{4}+\gamma_{5}
\end{array}\right]
$$

\subsection{Analysis of assembly deviation of threaded} fastening system

The deviation of the bolt fastening system is mainly reflected in the angle deviation $f_{\alpha}$ and position deviation $f_{\Sigma}$ between the actual position and the ideal position of the central axis of the fastening bolt. The main influencing factors are the position and verticality of the fastening bolt mounting hole on the cutter holder, clearance between shaft and hole, etc. To be consistent with the final test model, the disc cutter system model whose size is $1 / 3$ of the actual size is analyzed.

3.1.1 Establishing the dimensional chain of the threaded fastening system

Establish the dimension chain of the thread fastening system, as shown in Fig. 3. The thread fastening system assembly drawing and part tolerances, as shown in Fig. 4.

As shown in Fig. 3, let $F R_{\mathrm{b}}$ be the deviation of the fastening bolt in the reference coordinate system 0 . The 
system contains 6 internal connecting pairs (FE0, FE1), (FE0, FE2), (FE1', FE3), (FE2', FE4), (FE3', FE5), (FE4', FE5), and 4 external connecting pairs (FE1, FE1'), (FE2, FE2'), (FE3, FE3'), (FE4, FE4'). Because the load acts on the inner ring of the bearing and the load direction remain unchanged, there is an interference between the inner ring of the bearing and the fastening bolt, and there is a clearance between the outer ring of the bearing and the cutter holder. Clearances are between the bearing outer rings and the cutter holder, i.e. (FE1, FE1'), (FE2, FE2'). Set the dimension chain between the fastening bolt, bearing 1 and the cutter holder as dimension chain 1, i.e. (FE0 $\rightarrow$ $\left.\mathrm{FE} 1 \rightarrow \mathrm{FE}^{\prime} \rightarrow \mathrm{FE} 3 \rightarrow \mathrm{FE}^{\prime} \rightarrow \mathrm{FE} 5\right)$. Set the dimension chain between the fastening bolt, bearing 2 and the cutter holder as dimension chain 2, i.e. (FE0 $\rightarrow \mathrm{FE} 2 \rightarrow$ $\mathrm{FE} 2^{\prime} \rightarrow$ FE $4 \rightarrow$ FE4' $\rightarrow$ FE5).

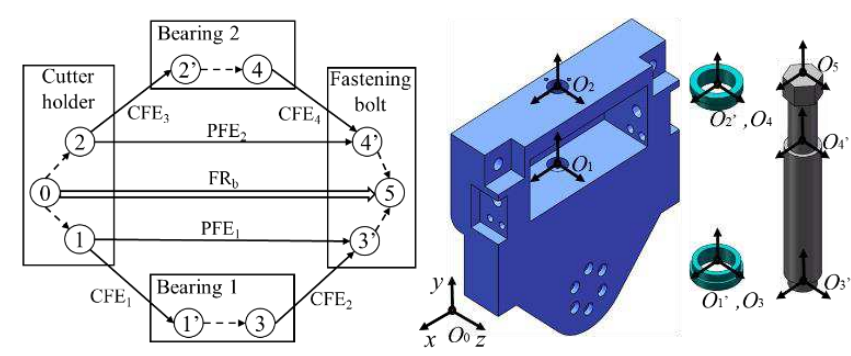

Fig. 3 Dimensional chains of threaded fastening system

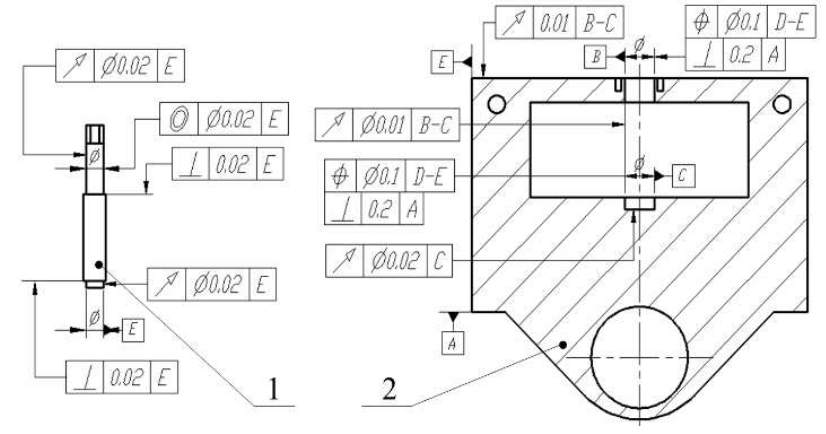

1- Fastening bolt, 2- Cutter holder

Fig. 4 Part tolerances of thread fastening system

3.1.2 Spinor representation of tolerance zone of the dimension chains

(1) Dimension chain 1

(1) Between bearing 1 and cutter holder

There are two parallel sub connecting pairs between bearing 1 and cutter holder. The lower end face of bearing 1 and the end face of the hole on the cutter holder form a face-to-face connecting pair, $T_{1}$. The clearance between the outer ring of bearing 1 and the hole's wall on the cutter holder is matched to form a cylindrical surface to cylindrical surface connecting pair, $T_{2}$.

According to the accuracy requirements, the tolerance field spinor of $T_{1}$ is as follows.

$$
\left\{\begin{array}{l}
u_{C 1}=w_{C 1}=\frac{0.022+0.015+0.02+0.02}{2}=0.0385 \\
v_{C 1}=0 \\
\alpha_{C 1}=\delta_{C 1}=\frac{0.022+0.015+0.02+0.02}{2.25}=0.0342 \\
\beta_{C 1}=0
\end{array}\right.
$$

So,

$$
T_{1}=\left[\begin{array}{llllll}
0.0385 & 0 & 0.0385 & 0.0342 & 0 & 0.0342
\end{array}\right]^{T}
$$

According to the accuracy requirements, the tolerance field spinor of $T_{2}$ is as follows.

$$
\left\{\begin{array}{l}
u_{P 2}=w_{P 2}=0 \\
v_{P 2}=0.02+0.02=0.0400 \\
\alpha_{P 2}=\delta_{P 2}=\frac{0.02+0.02}{10.667}=0.00375 \\
\beta_{P 2}=0
\end{array}\right.
$$

So,

$$
T_{2}=\left[\begin{array}{llllll}
0 & 0.0400 & 0 & 0.00375 & 0 & 0.00375
\end{array}\right]^{T}
$$

According to the solution method of the local parallel dimension chain[23], the union operation is performed on the three translation parameters of two parallel sub connecting pairs, and the intersection operation is performed on the three rotation parameters.

So,

$$
C F E_{1}=\left[\begin{array}{llllll}
0.0385 & 0.0400 & 0.0385 & 0.00375 & 0 & 0.00375
\end{array}\right]^{T}
$$

(2) Between bearing 1 and fastening bolt

There is an interference fit between bearing 1 and the fastening bolt. Therefore, only the surface connection pair composed of the upper-end face of bearing 1 and the shoulder end face of the fastening bolt is considered, and its tolerance field spinor is as follows. 


$$
\left\{\begin{array}{l}
u_{P 2}=w_{P 2}=0 \\
v_{P 1}=0.02+0.2+0.1=0.32 \\
\alpha_{P 1}=\delta_{P 1}=\frac{0.02+0.2+0.1}{8.75}=0.0366 \\
\beta_{P 1}=0
\end{array}\right.
$$

So,

$$
C F E_{2}=\left[\begin{array}{llllll}
0 & 0.320 & 0 & 0.0366 & 0 & 0.0366
\end{array}\right]^{T}
$$

Then, the Jacobian-Torsor model of dimension chain 1 is as follows.

$$
P F E_{1}=\left[\begin{array}{l}
J_{C F E 1} J_{I F E 3} \\
J_{C F E 2}
\end{array}\right]^{T}\left[\begin{array}{l}
{[C F E 1]} \\
{[I F E 3]} \\
{[C F E 2]}
\end{array}\right]=\left[\begin{array}{c}
0.0385 \\
0.36 \\
0.0385 \\
0.0416 \\
0 \\
0.0416
\end{array}\right]
$$

Where,

$$
\begin{aligned}
& J_{C F E 1}=\operatorname{diag}(1,1,1,1,1,1)_{C F E 1} \\
& J_{C F E 2}=\operatorname{diag}(1,1,1,1,1,1)_{C F E 2} \\
& J_{I F E 3}=\operatorname{diag}(1,1,1,1,1,1)_{I F E 3} \\
& {[\text { IFE3 }]=0}
\end{aligned}
$$

(2) Dimension chain 2

(1) Between bearing 2 and cutter holder

There is a clearance fit between the outer ring of bearing 2 and the mounting hole of the cutter base, so it forms a cylindrical to cylindrical connecting pair. According to the accuracy requirements, its tolerance field spinor is as follows.

$$
\left\{\begin{array}{l}
u_{C 3}=w_{C 3}=\frac{0.022+0.015+0.02+0.02}{2}=0.0385 \\
\alpha_{C 3}=\delta_{C 3}=\frac{0.022+0.015+0.02+0.02}{2.5}=0.0308
\end{array}\right.
$$

$$
C F E_{3}=\left[\begin{array}{llllll}
0.0385 & 0 & 0.0385 & 0.0308 & 0 & 0.0308
\end{array}\right]^{T}
$$

(2) Between bearing 2 and fastening bolt

There is an interference fit between the inner ring of bearing 2 and the fastening bolt. Therefore, only the surface connection pair composed of the lower end face of bearing 2 and the end face of the shoulder of the fastening bolt is considered. According to the accuracy requirements, its tolerance field spinor is as follows.

$$
\left\{\begin{array}{l}
u_{C 2}=w_{C 2}=\frac{0.2+0.1+0.02}{2}=0.160 \\
\alpha_{C 2}=\delta_{C 2}=\frac{0.2+0.1+0.02}{1.25}=0.256
\end{array}\right.
$$

So,

$$
C F E_{4}=\left[\begin{array}{llllll}
0.160 & 0 & 0.160 & 0.256 & 0 & 0.256
\end{array}\right]^{T}
$$

So, the Jacobian-Torsor model of dimension chain 2 is as follows.

$$
P F E_{2}=\left[\begin{array}{l}
J_{C F E 3} \\
J_{\text {IFE5 }} \\
J_{\text {CFE } 4}
\end{array}\right]^{T}\left[\begin{array}{l}
{[\text { CFE3 }]} \\
{[\text { IFE5 }]} \\
{[\text { CFE4 }]}
\end{array}\right]=\left[\begin{array}{c}
0.199 \\
0.0400 \\
0.199 \\
0.261 \\
0 \\
0.261
\end{array}\right]
$$

Where,

$$
\begin{aligned}
& J_{C F E 3}=\operatorname{diag}(1,1,1,1,1,1)_{C F E 3} \\
& J_{C F E 4}=\operatorname{diag}(1,1,1,1,1,1)_{C F E 4} \\
& J_{I F E 5}=\operatorname{diag}(1,1,1,1,1,1)_{\text {IFE } 5} \\
& {[\text { IFE } 5]=0}
\end{aligned}
$$

To sum up, variations of torsor models of the thread fastening system are shown in Table 1.

So,

Table 1 Variations of torsor models of the thread fastening system

\begin{tabular}{cc}
\hline Tolerance zone \\
\hline Parameter
\end{tabular}




\subsubsection{Establish Jacobian-Torsor model}

The Jacobian-Torsor model of the local coordinate system 5 of the fastening bolt in the reference coordinate system 0 of the cutter base is established. The local coordinate origin $O_{5}$ is the center of the upper end face of the fastening bolt.

$$
\begin{gathered}
T_{a 11}=\left[\begin{array}{llllll}
0.304 & 0 & 0.304 & 0.00496 & 0 & 0.00496
\end{array}\right]^{T} \\
J_{O_{0}}^{O_{5}}=\left[\begin{array}{cccccc}
1 & 0 & 0 & 0 & 0 & -35.75 \\
0 & 1 & 0 & 0 & 0 & 0 \\
0 & 0 & 1 & 35.75 & 0 & 0 \\
0 & 0 & 0 & 1 & 0 & 0 \\
0 & 0 & 0 & 0 & 1 & 0 \\
0 & 0 & 0 & 0 & 0 & 1
\end{array}\right] \\
F R_{b}=J_{O_{0}}^{O_{5}}\left[T_{a 11}\right]=\left[\begin{array}{c}
0.127 \\
0 \\
0.481 \\
0.00496 \\
0 \\
0.00496
\end{array}\right]
\end{gathered}
$$

\subsection{Calculation of assembly deviation between disc} cutter and cutter holders

There is a clearance between the disc cutter mounting hole on the cutter holder and the disc cutter shaft, and the two are fastened by bolts. The fit deviation will affect the distance and posture between the two fastening bolts. The deviation is shown in Fig. 5. Due to the symmetry of the new integrated disc cutter system, only the right-side matching pair is calculated. The local coordinates are shown in Fig. 6.

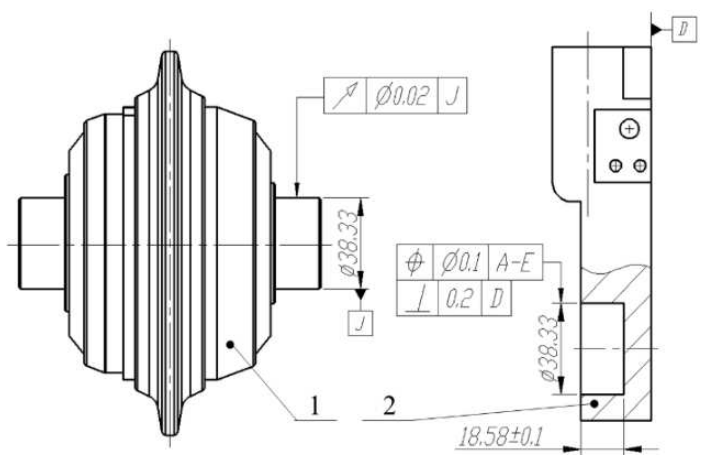

1- Disc cutter, 2- Cutter holder

Fig. 5 Tolerance of disc cutter and cutter holder

There are two parallel sub-connection pairs between the mounting hole on the cutter holder and the disc cutter. The cylindrical surface-cylindrical surface connection pair formed by the inner wall of the mounting hole and the shaft surface of the disc cutter, set to $T_{3}$. And the face-face connection pair is formed by the end face of the mounting hole and the end face of the disc cutter shaft, set to $T_{4}$.

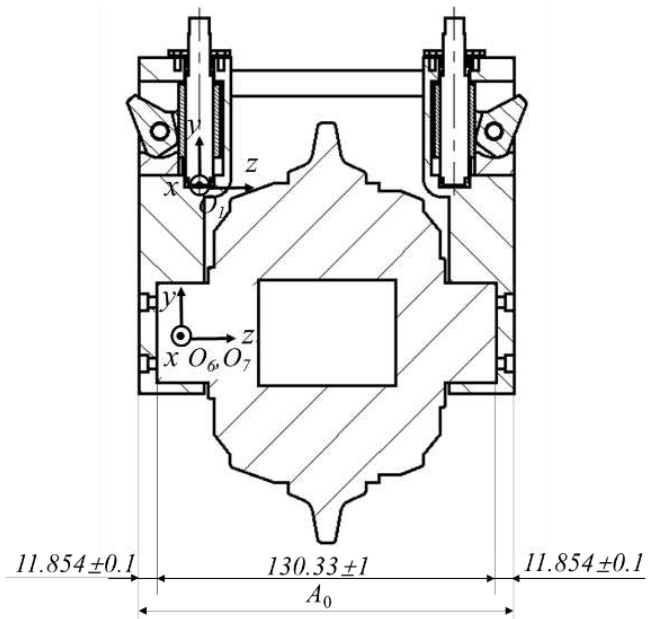

Fig. 6 Local coordinates of cutter holder and disc cutter

According to the accuracy requirements, the tolerance field spinor of $T_{3}$ is as follows.

$$
\left\{\begin{array}{l}
u_{C 6}=w_{C 6}=\frac{0.033+0.021+0.1+0.2}{2}=0.177 \\
v_{C 6}=0 \\
\alpha_{C 6}=\delta_{C 6}=\frac{0.033+0.021+0.1+0.2}{13.94}=0.0254 \\
\beta_{C 6}=0
\end{array}\right.
$$

So,

$$
T_{3}=\left[\begin{array}{llllll}
0.177 & 0 & 0.177 & 0.0254 & 0 & 0.0254
\end{array}\right]^{T}
$$

According to the accuracy requirements, the tolerance field spinor of $T_{4}$ is as follows.

$$
\left\{\begin{array}{l}
u_{P 5}=w_{P 5}=0 \\
v_{P 5}=0.02+0.02=0.0400 \\
\alpha_{P 5}=\delta_{P 5}=\frac{0.02+0.02}{28.75}=0.00139 \\
\beta_{P 5}=0
\end{array}\right.
$$

So,

$$
T_{4}=\left[\begin{array}{llllll}
0 & 0.0400 & 0 & 0.00139 & 0 & 0.00139
\end{array}\right]^{T}
$$

Same with CFR1. 


$$
C F R_{7}=\left[\begin{array}{llllll}
0.177 & 0.0400 & 0.177 & 0.00139 & 0 & 0.00139
\end{array}\right]^{T}
$$

The Jacobian-Torsor model of the local coordinate system 7 of the disc cutter in the local coordinate system 1 of the cutter holder is established. According to the relative position of the two, The Jacobian matrix is as follows.

The Jacobian-Torsor model is as follows.

$$
F R_{t}=J_{C F E 7}\left[C F E_{7}\right]=\left[\begin{array}{c}
0.117 \\
0.0332 \\
0.237 \\
0.00139 \\
0 \\
0.00139
\end{array}\right]
$$

Where,

$$
J_{C F E 7}=\left[\begin{array}{cccccc}
1 & 0 & 0 & 0 & 4.875 & -43.275 \\
0 & 1 & 0 & -4.875 & 0 & 0 \\
0 & 0 & 1 & 43.275 & 0 & 0 \\
0 & 0 & 0 & 1 & 0 & 0 \\
0 & 0 & 0 & 0 & 1 & 0 \\
0 & 0 & 0 & 0 & 0 & 1
\end{array}\right]_{C F E 7}
$$

Then, the assembly deviation between disc cutter and cutter holder is calculated by the extreme value method, and the tolerance is shown in Fig. 6.

According to the loop method, all dimensions are added rings. So,

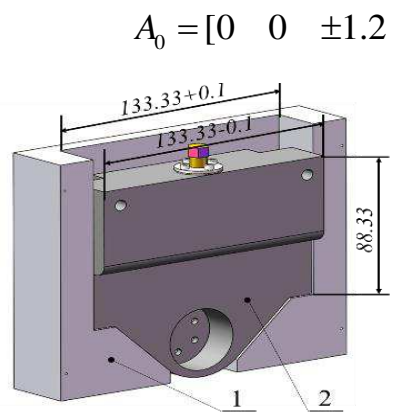

(a) Dimensions of cutter holder and cutter box

1- Cutter box, 2- Cutter holder

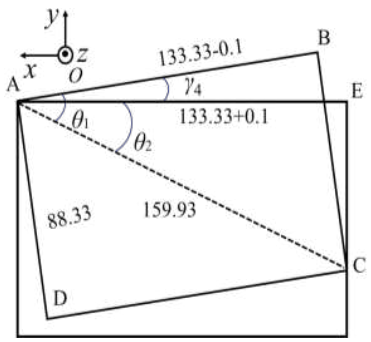

(b) The geometric model of angle deviation between cutter

holder and cutter box
Fig. 7 assembly deviation between cutter holders and cutter box

\subsection{Calculation of assembly deviation between cutter holders and cutter box}

The tolerance of the cutter holder and cutter box is shown in Fig. 7(a). Clearance fit between cutter holder and cutter box may cause displacement deviation and angle deviation. The geometric model of angle deviation is shown in Fig. 7(b).

According to geometric relations, the maximum deviation between the cutter holder and the cutter box is as follows.

$$
F R_{h}=\left[\begin{array}{llllll}
0.1 & 0 & 0 & 0 & 0 & 0.132
\end{array}\right]^{T}
$$

\subsection{Calculation of welding deviation between cutter} box and cutter head

The cutter box of the new integrated disc cutter system needs to be welded in the directional mounting hole on the cutter head. The welding seam will cause deviations in the position of the cutter box on the cutter head, which will affect the position of the cutter box in the global coordinate system. The overall dimensions of the cutter box are shown in Fig. 8(a).

Assuming that in the global coordinate system 0 , the gap between the cutter box and the mounting hole on the cutter head in the $x$-axis direction is $x$, and the gap in the $z$-axis direction is $z$. The geometric model is shown in Fig. 8(b).

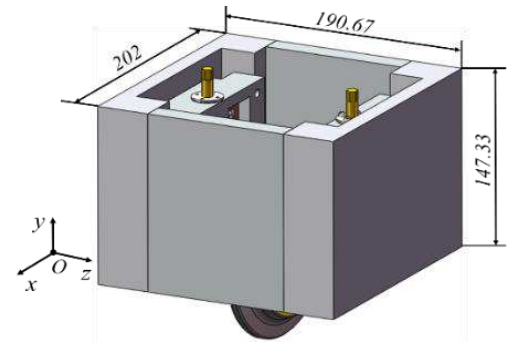

(a) Overall dimensions of the cutter box

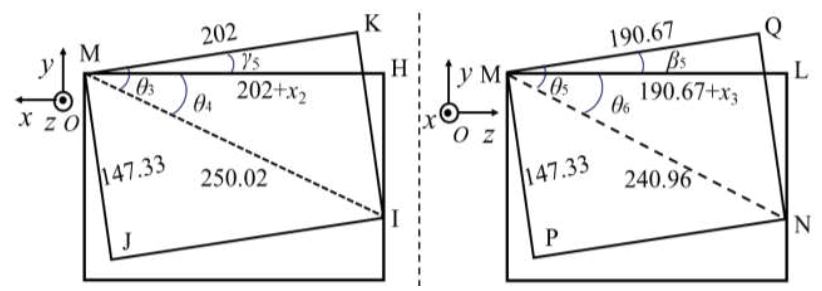

(b) The geometric model of angle deviation between the cutter box and the mounting hole of the cutter head

Fig. 8 The welding deviation between cutter box and cutter head

In the actual situation, the installation gap between the installation hole on the cutter head and the cutter box is about $10 \mathrm{~mm}$. In the scaled-down disc cutter 
model, the installation gap is calculated as $3.3 \mathrm{~mm}$, that is, $x_{2 \max }=x_{3 \max }=3.3 \mathrm{~mm}$. So, the calculation of welding deviation between the cutter box and the cutter head is as follows.

$$
N=\left[\begin{array}{llllll}
0 & 0 & 0 & 0 & 1.302 & 1.304
\end{array}\right]^{T}
$$

\subsection{Calculation of comprehensive deviation of fastening bolt}

Through the above analysis and calculation, the various Jacobian-Torsor models in Table 2 can be obtained.

Table 2 Jacobian-Torsor models of the deviation of each part of the new integrated disc cutter system

\begin{tabular}{|c|c|c|}
\hline Parameters & Physical significance & Tolerance zone \\
\hline$F R_{\mathrm{b}}$ & $\begin{array}{l}\text { Assembly deviation between fastening bolt and } \\
\text { cutter holder }\end{array}$ & 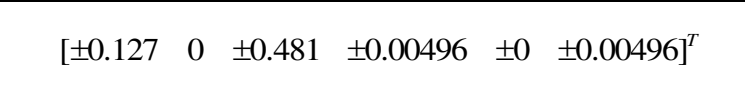 \\
\hline$F R_{\mathrm{t}}$ & $\begin{array}{l}\text { Assembly deviation between disc cutter and } \\
\text { cutter holders }\end{array}$ & {$\left[\begin{array}{llllll} \pm 0.117 & \pm 0.0332 & \pm 0.237 & \pm 0.00139 & \pm 0 & \pm 0.00139\end{array}\right]^{T}$} \\
\hline$A_{0}$ & $\begin{array}{l}\text { The deviation between cutter holders and disc } \\
\text { cutter in the direction of cutter shaft }\end{array}$ & {$\left[\begin{array}{llllll}0 & 0 & \pm 1.20 & 0 & 0 & 0\end{array}\right]^{T}$} \\
\hline$F R_{\mathrm{h}}$ & $\begin{array}{l}\text { Assembly deviation between cutter holders and } \\
\text { cutter box }\end{array}$ & {$\left[\begin{array}{llllll} \pm 0.1 & 0 & 0 & 0 & 0 & \pm 0.132\end{array}\right]^{T}$} \\
\hline$N$ & $\begin{array}{l}\text { Welding deviation between cutter box and cutter } \\
\text { head }\end{array}$ & {$\left[\begin{array}{llllll}0 & 0 & 0 & 0 & \pm 1.302 & \pm 1.304\end{array}\right]^{T}$} \\
\hline
\end{tabular}

When calculating the comprehensive deviation of the integrated disc cutter system, each spinor parameter takes the maximum value. and the direction of each local coordinate system is consistent with the global coordinate system. The comprehensive spinor of the fastening bolt system of the new integrated disc cutter system can be obtained, as shown in Eq. 35 .

$$
E=\left[\begin{array}{c} 
\pm 0.344 \\
\pm 0.0335 \\
\pm 1.918 \\
\pm 0.00635 \\
\pm 1.302 \\
\pm 1.442
\end{array}\right]
$$

\subsection{Comprehensive deviation envelope circle of fastening bolt head}

Based on the analysis of the assembly deviation of the fastening bolt system, the possible area of the hexagon head of the fastening bolt is studied. The actual fastening bolts of the disc cutter system are nonstandard parts, which specification is M32. In the model reduced by 1:3, use standard M10 bolts.

The possible area of the hexagon head of the fastening bolt is a circular area with the ideal position of the bolt central axis as the center and $R$ as the radius. The comprehensive deviation envelope circle is shown in Fig. 9.

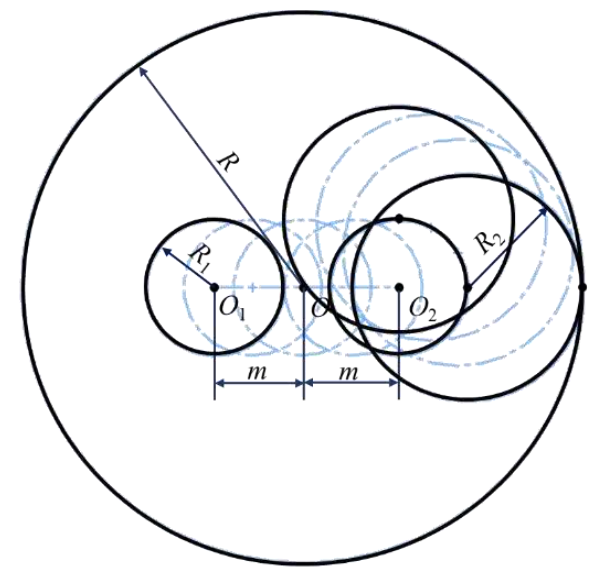

Fig. 9 The comprehensive deviation envelope circle of the fastening bolt head of the new integrated disc cutter system

$$
R=m+R_{1}+R_{2}
$$

Where $m$ is the assembly deviation between the cutter holder and the disc cutter in the direction of the disc cutter's central axis, $m= \pm 0.6 \mathrm{~mm}$.

$R_{1}$ is the larger of the components $u_{1}$ and $\left(w_{1}-m\right)$ of the composite deviation $F R_{\mathrm{b}}$ of the disc cutter system, $R_{1}=\max \left\{u_{1}, w_{1}-m\right\}=1.351 \mathrm{~mm}$.

$R_{2}$ is the circumscribed radius of the hexagon head of the fastening bolt, $R_{2}=9.24 \mathrm{~mm}$.

So, $R=m+R_{1}+R_{2}=11.191 \mathrm{~mm}$. 


\subsection{The measurement of the position deviation of} the fastening bolts of the new integrated disc cutter system

Use the coordinate measuring machine to measure the scaled-down model of the new integrated disc cutter system. Establish the coordinate system as shown in Fig. 10. The position and angle deviations in the $y$-axis direction are not considered. The ideal position and angle deviation of the two fastening bolts' center axis are as follows.

$$
\begin{aligned}
& {\left[\begin{array}{llllll}
101.100 & 0 & 36.700 & 0 & 0 & 0
\end{array}\right]^{T}} \\
& {\left[\begin{array}{llllll}
101.100 & 0 & 154.01 & 0 & 0 & 0
\end{array}\right]^{T}}
\end{aligned}
$$

Ignore the welding deviation between the cutter box and the cutter head. The theoretical position deviation of fastening bolts is as follows.

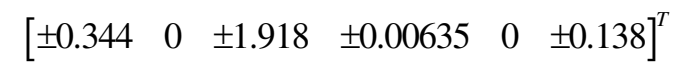

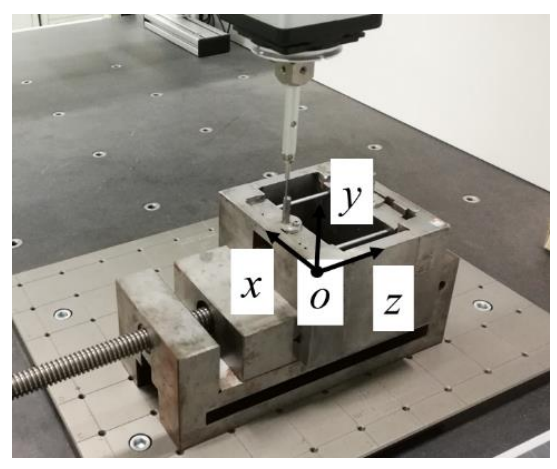

Fig. 10 Reference coordinate system

After measuring the coordinate position of each point of the disc cutter system, according to the spatial coordinate value of these points, through mathematical calculations, the actual position and angle deviations of the two fastening bolts center axis can be obtained.

$$
\begin{aligned}
& {\left[\begin{array}{llllll}
100.875 & 0 & 37.804 & 0.00517 & 0 & 0.131
\end{array}\right]^{T}} \\
& {\left[\begin{array}{llllll}
101.287 & 0 & 153.497 & 0.00419 & 0 & 0.145
\end{array}\right]^{T}}
\end{aligned}
$$

Compared to the ideal state, the deviation of position and angle deviations of the two fastening bolts center axis are as follows.

$$
\left[\begin{array}{llllll}
+0.225 & 0 & +1.104 & 0.00517 & 0 & 0.131
\end{array}\right]^{T}
$$

$$
\left[\begin{array}{llllll}
-0.187 & 0 & -0.513 & 0.00419 & 0 & 0.145
\end{array}\right]^{T}
$$

The results show that the angular deviation of the fastening bolt 2 winds around the $z$-axis is more than $5 \%$ of the theoretical calculation range. The rest are within the theoretical range. Therefore, the deviation analysis method is reasonable.

\section{Design of the end-effector of supporting cutter-changing robot}

For the above-mentioned integrated disc cutter, the end-effector of the robot has been designed, that is, a set of universal wrench for fastening bolts. There is a relative position deviation between the axis of the robot end-effector and the axis of the fastening bolt (Deviation 1), and there is a phase deviation between the head of the fastening bolt and the universal wrench (Deviation 2). For these two kinds of deviations, the end-effector of the cutter-changing robot needs to complete the radial and circumferential automatic positioning.

\subsection{Overall structure design of end effector of tool changing robot}

The structure of the cutter-changing robot endeffector is shown in Fig. 11.

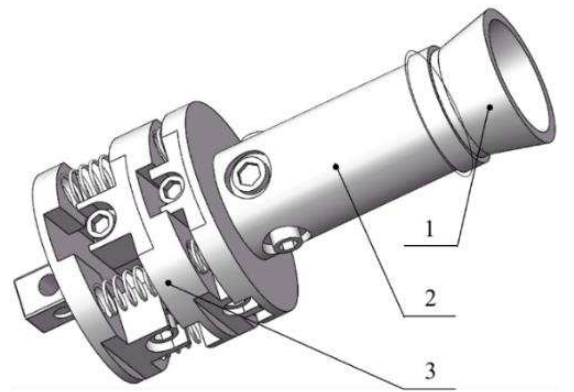

1- The guiding mechanism, 2- The universal wrench, 3- The flexible connecting mechanism

Fig. 11 The end-effector of the cutter-changing robot

Aiming at Deviation 1, a cone-shape guiding mechanism is designed. The guiding mechanism is similar to the horn type. When working, the head of the disc cutter fastening bolt enters from the trumpetshaped big end of the guiding mechanism, and slides along its inner wall to the small end of the guiding mechanism, and reaches the main body of the universal wrench. The guiding mechanism can contain a certain 
degree of relative position deviation between the axis of the universal wrench and the axis of the fastening bolt through the conical structure.

When the universal wrench at the end of the robot rotates the bolt, the above Deviation 1 will cause the universal wrench rotation eccentrically. Therefore, a flexible terminal connecting mechanism is designed. The mechanism consists of two universal joints, which can achieve a certain degree of eccentric transmission.

Aiming at the above Deviation 2, a universal wrench is designed. The universal wrench is composed of a sleeve and multiple steel bars. When working, the steel bars in contact with the end face of the bolt are pressed down, and the steel bars around the bolt fix the head of the bolt to apply torque to the bolt. The mechanism can accommodate fastening bolts at any angle.

\subsection{Parametric design of end-effector of supporting cutter-changing robot}

According to the assembly deviation analysis results of the new integrated disc cutter system, the parametric design of the universal wrench is carried out.

\subsubsection{Parametric design of the guiding mechanism}

The basic parameters of the guiding mechanism include the diameter of the big end $a$, the diameter of the small end $b$, and the length $l$, as shown in Fig. 12(a). (a) Parameters of the guiding mechanism

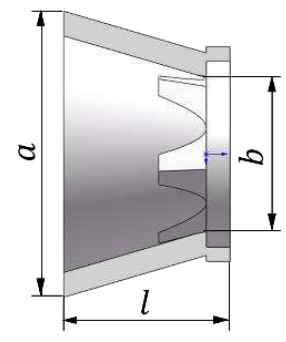

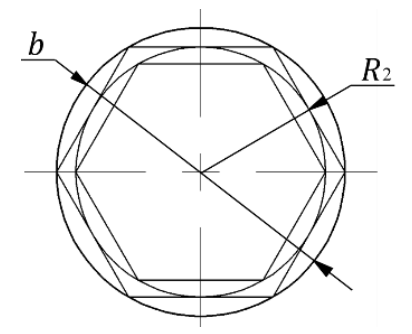

(b) Diameter of the guiding mechanism's small end
Fig. 12 Parametric design of the guiding

\section{mechanism}

(1) The diameter of the big end $a$

The diameter of the big end of the guiding mechanism shall be greater than the diameter of the comprehensive deviation envelope circle of the fastening bolt head of the disc cutter system, $D_{3}$, taking the safety factor of 1.2, then, $a=1.2 D_{3}=16.8594 \mathrm{~mm}$ 。

(2) The diameter of the small end $b$

The diameter of the small end of the guiding mechanism is equal to the diameter of the circumscribed circle of the regular hexagon with the circumscribed circle of the fastening bolt head as the inscribed circle, as shown in Fig. 12(b).

$$
b=\frac{2 R_{2}}{\cos 30^{\circ}}
$$

$R_{2}$ is the circumscribed radius of the fastening bolt head, $R_{2}=9.24 \mathrm{~mm}$.

(3) The length of the guiding mechanism $l$

The length of the guiding mechanism shall not affect the swing angle of the universal joint of the flexible connecting mechanism.

\subsubsection{Parametric design of the universal wrench}

The basic structural parameters of the universal wrench include the diameter of the circumscribed circle of the hexagonal inner hole $c$, the diameter of the steel bar $e$, the working depth of the steel bar $f$, the telescopic distance of the steel bar $h$, the length of the steel bar $g$, the height of the base $i$, the total length of the universal wrench $L$, as shown in Fig. 13.

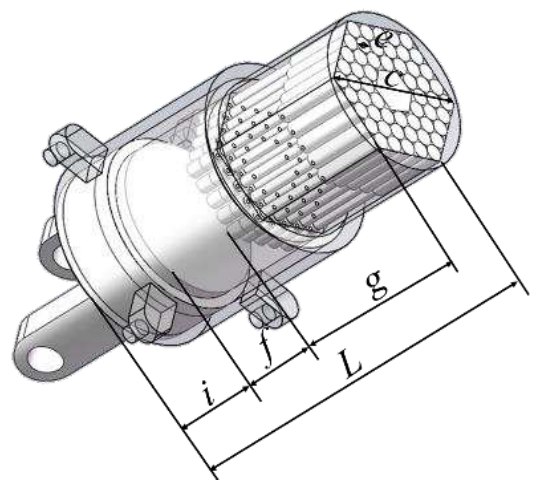

Fig. 13 Structural parameters of the universal wrench

(1) The diameter of the circumscribed circle of the hexagonal inner hole $c$

The diameter of the circumscribed circle of the hexagonal inner hole of the universal wrench $c$ is equal to the diameter of the small end of the guiding mechanism, $b$.

$$
c=b=\frac{2 R_{2}}{\cos 30^{\circ}}
$$

(2) The diameter of the steel bar $e$

The diameter of the steel bar should satisfy that the universal wrench can clamp the bolt at any angle, as shown in Fig. 14. It can be obtained from geometric 
relations

$$
e=\frac{R_{2}}{\left(3+\tan 30^{\circ}\right) \times \cos 30^{\circ}}
$$

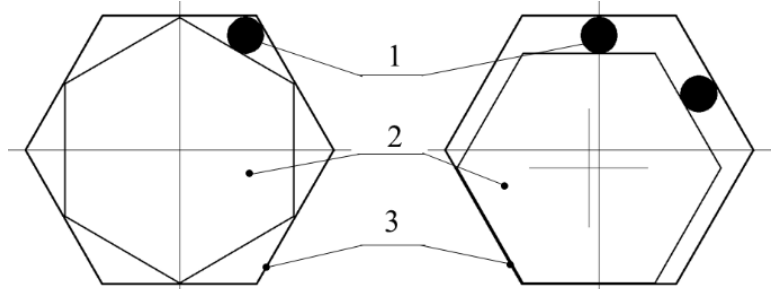

1- The steel bar, 2- The bolt, 3- The guiding mechanism's small end

Fig. 14 Matching relation between steel bars and bolt

(3) The working depth of the steel bar $f$

The working depth of the steel bar refers to the distance that the hexagonal head of the disc cutter system fastening bolt pushes the telescopic steel bar.

The working depth of the steel bar should be slightly less than the length of the bolt head. Set the length of the fastening bolt head of the scaled-down disc cutter system model as $l_{\mathrm{B}}, l_{\mathrm{B}}=6.4 \mathrm{~mm}$.

$$
f=l_{B}-2
$$

(4) The telescopic distance of the steel bar $h$

The retractable distance of the steel bar shall be slightly greater than the working depth of the steel bar, f.

$$
h=f+2
$$

(5) The length of the steel bar $g$

The steel bar includes the following three parts, the front part for clamping the bolt head, the back part that is fixed on the sleeve, and the middle part between them. Its lengths are set as $g_{1}, g_{2}$, and $g_{3}$ respectively, as shown in Fig. 15.

The $g_{1}$ should be slightly longer than the working depth of the steel bar, $f$.

$$
g_{1}=f+2
$$

The $g_{2}$ shall be slightly longer than the working depth of the steel bar, $f$, and the sum of the installation thickness of the steel bar $p$.

$$
g_{2}=f+p+2
$$

The $g_{3}$ shall ensure that the steel bar is stably fixed and less than the working depth of the steel bar $f$.

So,

$$
g=g_{1}+g_{2}+g_{3}=2 f+p+4+g_{3}
$$

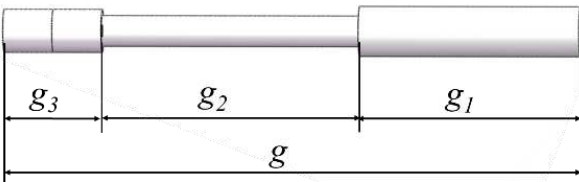

Fig. 15 Component of steel bar

(6) The height of the base $i$

The height of the base shall ensure sufficient strength requirements.

(7) The total length of the universal wrench $L$

The length of the universal wrench includes the length of the steel bar $g$, the working depth of the steel bar $f$, and the height of the limit base $i$.

$$
L=g+f+i
$$

4.2.3 Parametric design of the flexible connecting mechanism

The flexible connecting mechanism consists of two universal joints. Let the rod length of universal joint 1 be $s$ and the angle be $\varepsilon$. Let the rod length of universal joint 2 be $t$ and the angle be $\mu$. As shown in Fig. 16 .

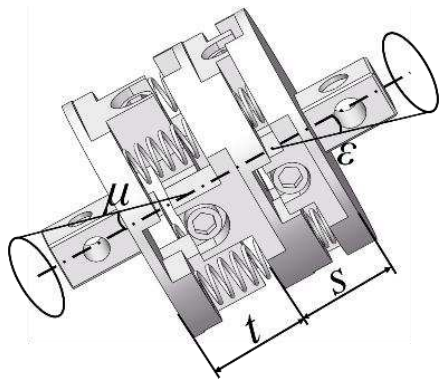

Fig. 16 Parameters of the flexible connecting mechanism

When the universal wrench is butted with the fastening bolt, the deviation includes radial deviation $z$ and angular deviation $\theta_{1}$ 。

$$
\begin{gathered}
Z=R_{1}+L+m \\
\theta_{1}=\max \{\alpha, \beta\}+\theta_{y}
\end{gathered}
$$

When the universal wrench is docked with the fastening bolt, the fastening bolt enters the guiding mechanism. Due to the radial deviation and the angle deviation, the axes of the two are not collinear and not 
parallel, and the guiding mechanism will rotate around the universal joint 1 by an angle of $\theta_{2}$. During the process of the universal wrench jamming the bolt, the flexible connecting mechanism rotates to eliminate this angle deviation.

(1) Determination of the rotation angle of the universal joint $1 \varepsilon$

The connecting process of the guiding mechanism and the fastening bolt is shown in Fig. 17, and the geometric relationship of each parameter is shown in Fig. 18.

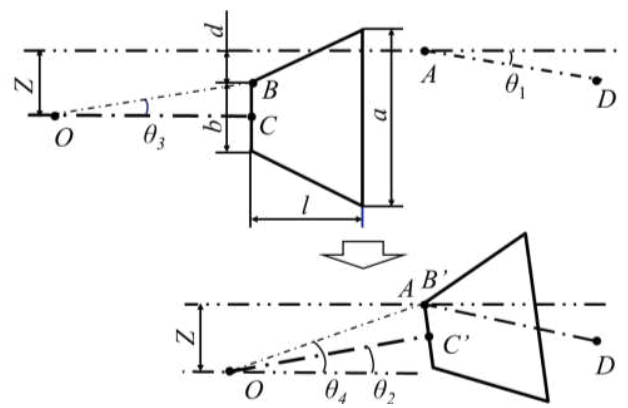

Fig. 17 The connecting process of the guiding mechanism and the fastening bolt

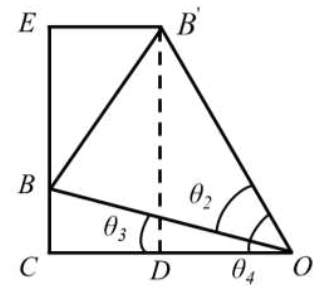

Fig. 18 The geometric relationship between the guiding mechanism and the fastening bolt

Suppose the distance from the small end section of the guiding mechanism to the center of rotation of the universal joint 1 is $O B=j$. The radius of the small end of the guide cone is $B C$. In the figure, $A D$ is the axis of the disc cutter tightening bolt.

To ensure reliability, take the safety factor of 1.2. And then, according to the geometry:

$$
\begin{aligned}
& \varepsilon=1.2 \times \max \left\{\theta_{1}, \theta_{2}\right\} \\
& =1.2 \times \max \left\{\begin{array}{l}
\max \{\alpha, \beta\}, \\
\arcsin \frac{\max \{\mathrm{u}, \mathrm{v}-\mathrm{m}\}+m}{j}-\arcsin \frac{R_{2}}{j \times \cos 30^{\circ}}
\end{array}\right\}
\end{aligned}
$$

(2) Determination of the rotation angle of the universal joint $2 \mu$

The process of connecting the end-effector of the cutter-changing robot with the bolt is shown in Fig. 19. In this process, the universal joint 2 rotates at an angle $\theta_{6}$, and the universal joint 1 rotates at an angle $\theta_{5}$ in a reverse direction so that the axis of the tightening bolt is parallel to the axis of the universal wrench. There are two cases of eccentricity and non-eccentricity, and the eccentric distance $k$ is the radius of the steel bar, that is, $k=e / 2$.

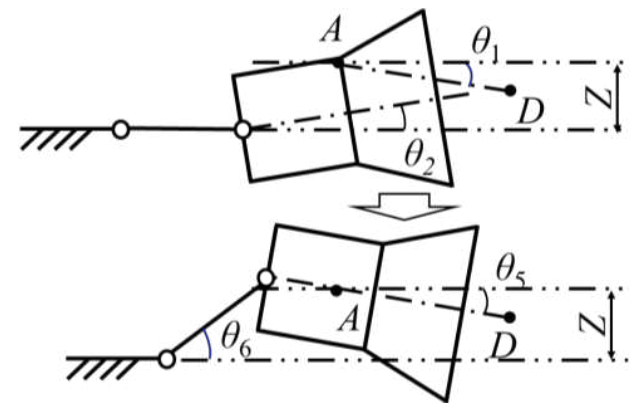

Fig. 19 The process of connecting the universal wrench with the bolt

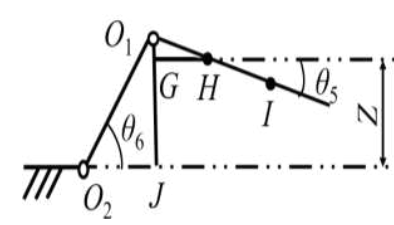

(a) No eccentricity

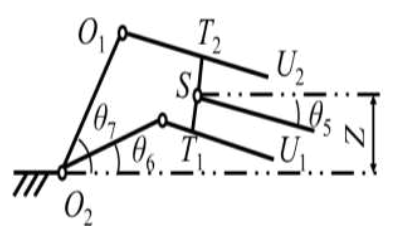

(b) Eccentricity
Fig.20 The schematic diagram of connecting the universal wrench with the bolt

Under the condition that there is no eccentricity between the universal wrench and the fastening bolt, the schematic diagram of the universal wrench and the bolt is shown in Fig. 20(a). When there is an eccentricity $k$ between the universal wrench and the fastening bolt, the schematic diagram of the universal wrench and the bolt is shown in Fig. 20(b).

In the figure, $H I$ is equal to the working depth of the universal wrench steel bar $f$. To ensure reliability, take the safety factor of 1.2 , then, 


$$
\mu=1.2 \times \max \left\{\theta_{6}, \theta_{6}^{\prime}\right\}=1.2 \times \max \left\{\begin{array}{l}
\arcsin \frac{(L+s-f) \times \sin (\max \{\alpha, \beta\})+\max \{\mathrm{u}, \mathrm{v}-\mathrm{m}\}+m}{t}, \\
\frac{(L+s+f) \sin (\max \{\alpha, \beta\})+\max \{\mathrm{u}, \mathrm{v}-\mathrm{m}\}+m-\frac{e}{2} \times \cos (\max \{\alpha, \beta\})}{t}
\end{array}\right\}
$$

\subsection{The simulation analysis}

The flexible connecting mechanism of the end effector of the cutter-changing robot is a weak component, so it is necessary to carry out finite element analysis on it. The flexible connecting mechanism is made of $20 \mathrm{CrMnTi}$, and the mechanical properties of 20CrMnTi are shown in Table 3. Grade $6.8 \mathrm{M} 10$ standard bolts are used in the scaled-down model of the new integrated TBM disc cutter. According to the mechanical design handbook, the required tightening torque of the bolts is $33 \sim 45 \mathrm{~N} \cdot \mathrm{m}$.

After necessary simplification of the flexible linkage model established by SolidWorks, it is imported into ANSYS/workbench, given materials and meshed. One end installed on the robot is fixed, and a moment of 45 $\mathrm{N} \cdot \mathrm{m}$ is applied on the other end. The analysis results are shown in Fig. 21.

Table 3 The mechanical properties of $20 \mathrm{CrMnTi}$

\begin{tabular}{ccccc}
\hline $\begin{array}{c}\text { Density } \\
\left(\mathrm{kg} / \mathrm{m}^{3}\right)\end{array}$ & $\begin{array}{c}\text { Elastic } \\
\text { modulus } \\
(\mathrm{GPa})\end{array}$ & $\begin{array}{c}\text { Poisson } \\
\text { ratio }\end{array}$ & $\begin{array}{c}\text { Tensile } \\
\text { strength } \\
(\mathrm{MPa})\end{array}$ & $\begin{array}{c}\text { Yield } \\
\text { strength } \\
(\mathrm{MPa})\end{array}$ \\
\hline 7800 & 207 & 0.25 & 1080 & 835 \\
\hline
\end{tabular}

(a) Distribution of stress

(b) Distribution of strain

Fig. 21 Finite element analysis results

Through the finite element analysis results, it can be found that under the action of $45 \mathrm{~N} \cdot \mathrm{m}$ moment, the maximum stress of the flexible connection mechanism is $323.49 \mathrm{MPa}$ and the maximum strain is $0.238 \mathrm{~mm}$, which is less than the required stress of its yield strength $835 \mathrm{MPa}$, which can meet the requirements.

\subsection{The universal wrench performance test}

To verify the designed end-effector of the cutterchanging robot in this paper whether it can meet the functional requirements, a model of cutter-changing robot end-effector for the scaled-down model of disc cutter system is made. The disc cutter system is simplified. The cutter-changing robot end-effector and the simplification device of the fastening bolt were mounted on the test bench as shown in Fig. 22. And docking tests were performed without deviations, a radial displacement deviation, and an angular deviation, as shown in Fig. 23.

The experimental results show that when there is a radial deviation of $3.55 \mathrm{~mm}$ between the cutter changing robot end-effector and the bolt, which accounts for $19.21 \%$ of the diameter of the circumscribed circle of the hexagon head of the bolt, the reduced scaled-down universal wrench can fully butt with the fastening bolt. The same result is obtained when there is an $11^{\circ}$ angle deviation between the two. which can meet the actual engineering requirements.

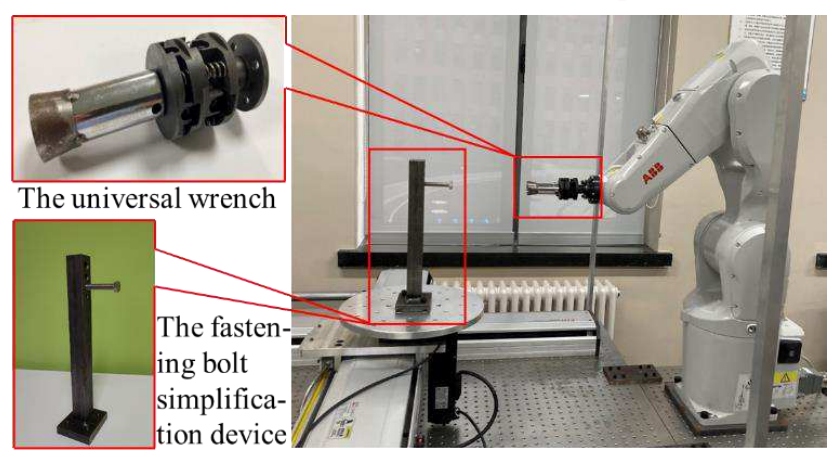

Fig. 22 The test bench 


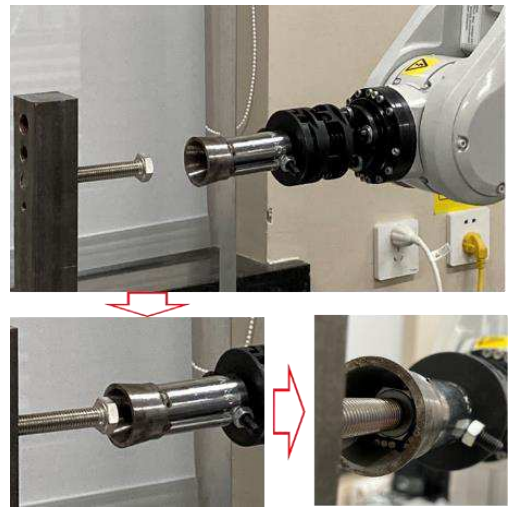

(a) The docking condition without deviation

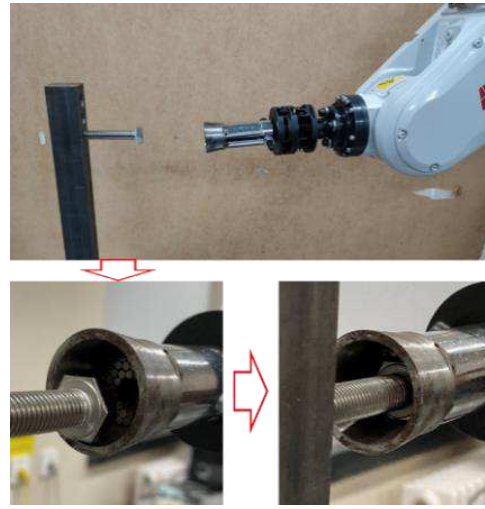

(b) The docking condition with radial deviation
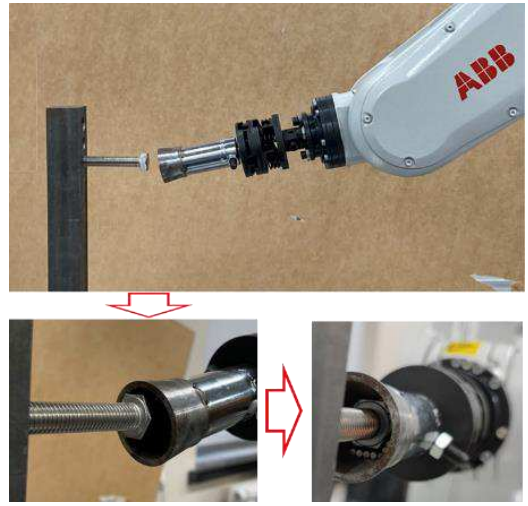

(c) The docking condition with angle deviation

Fig . 23 Docking tests

\section{Conclusion}

(1) In this paper, the improved Jacobian-Torsor model is used to analyze the deviation of a new integrated disc cutter system of TBM developed by our research group. The experiment results show that the deviation analysis method is reasonable and accurate. Among the 10 deviation components tested, only one exceeds the theoretical calculation range by $5 \%$.

(2) Based on deviation analysis, an end-effector of cutter-changing robot matched was designed, and a scaled-down sample was made for the test. The functions of each part of the universal wrench can meet the expected requirements. The scaled-down cutterchanging robot end-effector can accommodate a radial deviation of $3.55 \mathrm{~mm}$ or an angular deviation of $11^{\circ}$.

\section{Declarations}

Ethics approval and consent to participate

Not applicable.

\section{Consent for publication}

Not applicable.

\section{Availability of data and materials}

Readers can email the authors for data to support their findings.

\section{Competing interests}

The authors declare no competing fnancial interests.

\section{Funding}

This work was supported by the National key R \& D plan of China (Grant No. 2018YFB1306701) and the National Natural Science Foundation of China (Grant No. 51875076).

\section{Authors' contributions}

Hao Chen, Hao Li and Junzhou Huo completed the writing of the main part of the paper. Bowen Yang and Fan Yang have made contributions to the language of the paper. All authors read and approwed the final manuscriot.

\section{Acknowledgements}

The authors gratefully acknowledge Dr. Si Likun and our colleague Mr. Wang Shuo from the Key Laboratory of Precision and Special Processing, Ministry of Education, Dalian University of Technology, China, for their technical support during the experiments. And we thank our colleagues who provided insight and expertise that greatly assisted the research.

\section{Author Details}

${ }^{1}$ School of Mechanical Engineering, Dalian University of Technology, Dalian, 116024, China

\section{References}

[1] Camus, T. and G. Manacorda. European Project NeTTUN-Making it happen. in ITA WTC 2015 Congress and 41st General Assembly. 
2015. Lacroma Valamar Congress Center, Dubrovnik, Croatia.

[2] Derycke, J.N. and S. Rubrecht, Method for replacing a tunnel boring machine disk cutter, handling device and disk cutter suited to such a method. 2016: United States.

[3] Mansard, N., O. Khatib, and A. Kheddar, A unified approach to integrate unilateral constraints in the stack of tasks. IEEE Transactions on Robotics, 2009. 25(3): p. 670-685.

[4] Thomas Camus and S. Moubarak. Maintenance robotics in TBM tunnelling. in ISARC. Proceedings of the International Symposium on Automation and Robotics in Construction. 2015. IAARC Publications.

[5] Liu, Y., Typical Russian space docking mechanism and its characteristics. Spacecraft Engineering, 1994. 003(002): p. 33-38.

[6] Günther, H. and H.U. Knoop, Implication and usefulness of spacecraft servicing at the ISS using the pilot case of the large X-ray facility XEUS. Acta Astronautica, 2003. 53(4/10): p. 645-650.

[7] Zhang, L., et al., Analysis and Optimization of Force Transmissibility and Kinematic Performance of Low Impact Docking Mechanism. Manned Spaceflight, 2015. 21(5): p. 462-467.

[8] Chu, B., et al. Mechanism and analysis of a robotic bolting device for steel beam assembly. in ICCAS 2010. 2010. IEEE.

[9] Zhang, Q., et al. Development of Bolt Screwing Tool Based on Pneumatic Slip Ring. in 12th International Conference on Intelligent Robotics and Applications (ICIRA). 2019. Shenyang, PEOPLES R CHINA: Springer International Publishing Ag.

[10] Li, R.Y., et al., Unfastening of Hexagonal Headed Screws by a Collaborative Robot. Ieee Transactions on Automation Science and Engineering, 2020. 17(3): p. 1455-1468.

[11] DiFilippo, N.M. and M.K. Jouaneh, A System Combining Force and Vision Sensing for Automated Screw Removal on Laptops. Ieee Transactions on Automation Science and Engineering, 2018. 15(2): p. 887-895.

[12] Zhang, Q., et al., A novel approach for flexible manipulator conducting screwing task based on robot-environment contact classification. Proceedings of the Institution of Mechanical Engineers Part C-Journal of Mechanical Engineering Science, 2021. 235(8): p. $1357-1367$.

[13] Chen, H., et al., A comprehensive study of three dimensional tolerance analysis methods. Computer-Aided Design, 2014. 53: p. 1-13.

[14] Hong, Y. and T. Chang, A comprehensive review of tolerancing research. International Journal of Production Research, 2002. 40(11): p. $2425-2459$.

[15] Clement, A. and A. Riviere. Tolerancing versus nominal modeling in next generation CAD/CAM system. in Proceedings of 3rd CIRP Seminar on Computer Aided Tolerancing. 1993. ENS de Cachan, France:[sn].

[16] Clément, A. Theory and practice of 3-D tolerancing for assembly. in Proc. CIRP Int. Working Seminar on Computer-Aided Tolerancing. 1991.

[17] Laifa, M., W.B. Sai, and M. Hbaieb, Evaluation of machining process by integrating 3D manufacturing dispersions, functional constraints, and the concept of small displacement torsors. The International Journal of Advanced Manufacturing Technology, 2014. 71(5-8): p. 1327-1336.

[18] Davidson, J., A. Mujezinovic, and J. Shah, A new mathematical model for geometric tolerances as applied to round faces. J. Mech. Des., 2002. 124(4): p. 609-622.

[19] Jaishankar, L.N., J.K. Davidson, and J.J. Shah. Tolerance analysis of parallel assemblies using tolerance-maps ${ }^{\circledR}$ and a functional map derived from induced deformations. in ASME International Design Engineering Technical Conferences and Computers and Information in Engineering Conference (IDETC/CIE). 2013. Portland, OR: American Society of Mechanical Engineers.

[20] Barbero, B.R., J.P. Azcona, and J.G. Pérez, A tolerance analysis and optimization methodology. The combined use of 3D CAT, a dimensional hierarchization matrix and an optimization algorithm. The International Journal of Advanced Manufacturing Technology, 2015. 81(1): p. 371-385.

[21] Desrochers, A., W. Ghie, and L. Laperriere, Application of a unified Jacobian-torsor model for tolerance analysis. J. Comput. Inf. Sci. Eng., 2003. 3(1): p. 2-14. 
[22] Jin, S., et al., A small displacement torsor model for 3D tolerance analysis of conical structures. Proceedings of the Institution of Mechanical Engineers, Part C: Journal of Mechanical Engineering Science, 2015. 229(14): p. 2514-2523.

[23] Zeng, W.H., et al., A solution of worst-case tolerance analysis for partial parallel chains based on the Unified Jacobian-Torsor model. Precision Engineering-Journal of the International Societies for Precision Engineering and Nanotechnology, 2017. 47: p. $276-291$.

[24] Gao, J., K.W. Chase, and S.P. Magleby, Generalized 3-D tolerance analysis of mechanical assemblies with small kinematic adjustments. IIE transactions, 1998. 30(4): p. 367-377.

[25] Liu, W., et al., Mechanism Analysis of Deviation Sourcing and Propagation for Mechanical Assembly. Journal of Mechanical Engineering, 2012. 48(1): p. 156-168. 


\section{Supplementary Files}

This is a list of supplementary files associated with this preprint. Click to download.

- DemoVideo.mp4 